\title{
Maternal complications in molecularly confirmed diandric and digynic triploid pregnancies: single institution experience and literature review
}

\author{
D. Massalska ${ }^{1}$ D · J. Bijok ${ }^{1} \cdot$ A. Kucińska-Chahwan ${ }^{1} \cdot$ J. G. Zimowski ${ }^{2} \cdot K_{\text {. Ozdarska }}^{2} \cdot$ A. Raniszewska ${ }^{2} \cdot$ G. M. Panek $^{1}$. \\ T. Roszkowski ${ }^{1}$
}

Received: 9 January 2020 / Accepted: 12 March 2020 / Published online: 26 March 2020

(c) The Author(s) 2020

\begin{abstract}
Objectives Assessment of the maternal complications in molecularly confirmed diandric and digynic triploid pregnancies. Methods Sonographic features, biochemical results, and clinical presentation were analyzed. Beta-hCG level was controlled after diandric triploidy.

Results The study included nine diandric and twelve digynic triploid pregnancies at the mean gestational age at diagnosis of 14.9 and 18.0 weeks, respectively $(p=0.0391)$. Mean value of total-hCG was $979703.6 \mathrm{U} / \mathrm{ml}$ in diandric cases and 5455.4 $\mathrm{U} / \mathrm{ml}$ in digynic ones $(p<0.000)$. Maternal complications occurred in $88.9 \%$ of diandric triploid pregnancies, including: thecalutein cysts $(44.4 \%)$, hyperemesis gravidarum $(44.4 \%)$, symptomatic hyperthyreosis $(33.3 \%)$, early onset gestational hypertension $(22.2 \%)$ and vaginal bleeding (11.1\%). No case of proteinuria, preeclampsia or HELLP syndrome was observed. Only maternal complication observed in digynic triploidy was vaginal bleeding (50.0\%). The mean time of beta-hCG normalization after diandric triploid pregnancies was 84 days (range 11-142 days). No case of gestational trophoblastic neoplasia (GTN) was observed.

Conclusions Maternal complications (except for vaginal bleeding) are associated with diandric triploidy. The relatively low incidence of hypertensive maternal complications and their less severe course in our cohort may be attributed to the earlier prenatal diagnosis. The frequency of GTN after diandric triploidy may be lower than previously reported.
\end{abstract}

Keywords Diandric triploidy $\cdot$ Digynic triploidy $\cdot$ Maternal triploidy $\cdot$ Paternal triploidy $\cdot$ Partial hydatidiform mole Preeclampsia

\section{Introduction}

Triploidy is a lethal chromosomal abnormality resulting from an extra haploid chromosome set of maternal or paternal origin [18]. Pregnancy characteristics depend on parental contribution of the extra chromosomes. Typically diandric triploidy (type I) manifests with a relatively well-grown fetus with normal head size or microcephaly and enlarged

D. Massalska

diana_massalska@wp.pl

1 Department of Gynecologic Oncology and Obstetrics, Centre of Postgraduate Medical Education, Czerniakowska 231, 00-416 Warsaw, Poland

2 Department of Genetics, Institute of Psychiatry and Neurology, Sobieskiego 9, 02-957 Warsaw, Poland placenta of cystic appearance. Digynic triploidy (type II) presents with a severely growth-restricted fetus with a relative macrocephaly (asymmetric fetal growth restriction, FGR) and a very small non-cystic placenta. Moreover, variable structural defects may be observed [25].

The majority of triploid pregnancies is miscarried at an early developmental stage. Triploidy occurs in around $0.03 \%$ of pregnancies at 10-14 gestational weeks and $0.002 \%$ of pregnancies at 16-20 weeks [14, 20]. Triploidy may cause maternal complications such as preeclampsia, hyperthyroidism, gestational trophoblastic neoplasia or vaginal bleeding [17, 29, 38]. However, assessment of the risk for maternal complications depending on the parental origin of triploidy has not been performed to date. 


\section{Objectives}

The objective of the study was to assess the sonographic and biochemical characteristics as well as the risk for maternal complications in the molecularly confirmed diandric and digynic triploid pregnancies that survived beyond 13 gestational weeks.

\section{Material and methods}

\section{Material}

All consecutive singleton triploid pregnancies evaluated at our Ultrasound Department in the years 2017-2019 as a part of TRIPLOIDY PROJECT that survived beyond 13 gestational weeks were included. The study group consisted of 21 singleton triploid pregnancies-9 diandric (69, XXY; $n=7$ and $69, \mathrm{XXX} ; n=2)$ and 12 digynic (69, XXY; $n=1$ and $69, \mathrm{XXX} ; n=11)$.

\section{Methods}

We assessed the sonographic features, biochemical results and clinical presentation of triploid pregnancies examined at our institution in the years 2017-2019. All sonographic evaluations were performed by an experienced sonographer (T.R.). Gestational age was determined from the last menstrual period. Invasive diagnosis for genetic testing was carried out in all cases after informed consent.

The data regarding medical history and complications in present pregnancy (vaginal bleeding, vomiting, hypertension, proteinuria, hyperthyroidism and other abnormalities) were collected from a questionnaire filled by the patients. Urine and blood tests were performed (complete blood count $[\mathrm{CBC}]$, and creatinine, uric acid, urea, alanine and asparagine aminotransferase, lactate dehydrogenase, total-hCG, thyroid stimulating hormone [TSH], free thyroxine [fT4]) and blood pressure was measured. In order to exclude gestational trophoblastic neoplasms (GTN) after diandric triploid pregnancies complicated by molar changes the beta-hCG level was controlled every 2 weeks till normal values and a single control follow-up measurement in a month to confirm normal results was performed according to the protocol suggested by Coyle et al. [9].

\section{Tested samples}

21 DNA samples (chorionic villi; $n=11$ and amniocytes; $n=10$ ) with molecular diagnosis of triploidy and 42 matching blood samples from the parents were tested for parental origin with Quantitative Fluorescent Polymerase Chain Reaction (QF-PCR). The mean gestational age at genetic testing was 16.7 weeks (range 11.7-26.3 weeks).

\section{Quantitative fluorescent polymerase chain reaction (QF-PCR)}

QF-PCR reaction included amplification of 26 selected microsatellite sequences from chromosomes 13, 18, 21, $\mathrm{X}$ and $\mathrm{Y}$. The products were separated by capillary electrophoresis (3130 Genetic Analyser, Applied Biosystems, Waltham, MA, USA) and the results were analyzed by the use of GeneMapper Software 5.0 (Thermo Fisher Scientific, Waltham, MA, USA). The fetal DNA peaks of quantitative relation 1:2,2:1 and 1:1:1 were compared to the peaks from parental DNA, which allowed for establishment of the parental origin of the double microsatellite sequences (paternal or maternal origin of triploidy).

\section{Data analysis}

Statistical analysis was performed using STATA 12 (StataCorp). Descriptive statistics was presented with means and proportions. Student's test and Fisher's exact test were used to assess differences in continuous data.

\section{Literature review}

We conducted a literature review on the studies describing maternal complications in triploid pregnancies published between 1960 and 2019. Pubmed database (https://pubme d.com) was searched using the term "TRIPLOIDY" and all abstracts were viewed by two authors (DM and AKC). Only full text English-written manuscripts were included. Additionally, the references of all selected manuscripts were screened for subsequent reports. Discrepancies between authors were resolved through discussion, until consensus was reached.

\section{Results}

The study included nine diandric and twelve digynic triploid pregnancies diagnosed at a single institution in the period of three years. Genetic testing was performed at a mean gestational age of 14.9 weeks (range 11.7-17.6 weeks) in diandric cases and at 18.0 weeks (range 12.4-26.3 weeks) in digynic ones $(p=0.0391)$. The mean maternal age at diagnosis in diandric triploidy was 31.1 years and in digynic triploidy: 33.4 ( $p>0.05)$.

In all cases the indication for genetic testing was abnormal fetal ultrasound $(n=21)$. Sonographic abnormalities detected in diandric and digynic cases are presented in 
Table 1. In all diandric cases the placenta was cystic $(n=9)$ and in none of digynic $(n=12)$.

The mean total-hCG level at invasive testing in diandric cases was 979, 703.6 U/ml (range 382, 261.0-1, 636, 562.0 $\mathrm{U} / \mathrm{ml}$ ) compared to $5455.4 \mathrm{U} / \mathrm{ml}$ in digynic ones (range 1, $245.2-19,050.6 \mathrm{U} / \mathrm{ml})(p<0.000)$. CBC, creatinine, uric acid, urea and lactate dehydrogenase levels were normal in all diandric and all digynic cases. In two diandric cases alanine and asparagine aminotransferase levels were slightly increased $(2 / 9 ; 22.2 \%)$.

Maternal complications were observed in eight diandric triploid pregnancies $(8 / 9 ; 88.9 \%)$ compared to six digynic ones $(6 / 12 ; 50.0 \%)(p>0.05)$. Maternal complications in diandric triploid pregnancies included the following: thecalutein cysts: $(4 / 9 ; 44.4 \%)$, hyperemesis gravidarum: (4/9; $44.4 \%)$, symptomatic hyperthyreosis: $(3 / 9 ; 33.3 \%)$, early onset gestational hypertension: $(2 / 9 ; 22.2 \%)$ and vaginal bleeding: $(1 / 9 ; 11.1 \%)$ (Table 2$)$. No case of proteinuria, preeclampsia or HELLP syndrome was observed. The only maternal complication observed in digynic triploidy was vaginal bleeding, which occurred in six cases $(6 / 12 ; 50.0 \%)$.

The pregnancy outcome was known for all cases. The mean pregnancy duration was 16.3 gestational weeks in diandric cases (range 14.1-19.6 weeks) and 19.3 weeks in digynic cases (range 13.4-30.3) $(p=0.0613)$. Seven diandric and seven digynic triploid pregnancies were terminated;
Table 1 Sonographic abnormalities in molecularly confirmed diandric and digynic triploidy

\begin{tabular}{|c|c|c|}
\hline Sonographic abnormalities & $\begin{array}{l}\text { Diandric triploid pregnancies } \\
(n=9)\end{array}$ & $\begin{array}{l}\text { Digynic triploid pregnancies } \\
(n=12)\end{array}$ \\
\hline Fetal growth restriction (FGR) & $0(0 \%)$ & $\begin{array}{l}11(100 \%) \\
\text { All asymmetrical }\end{array}$ \\
\hline Oligohydramnios & $1(11.1 \%)$ & $1(8.3 \%)$ \\
\hline \multicolumn{3}{|l|}{ Structural defects } \\
\hline Structural defects & $8(88.9 \%)$ & $10(83.3 \%)$ \\
\hline Multiple structural defects & $8(88.9 \%)$ & $7(58.3 \%)$ \\
\hline Central nervous system defects & $5(55.6 \%)$ & $5(41.7 \%)$ \\
\hline Ventriculomegaly & 1 & 1 \\
\hline Enlarged cysterna magna & 2 & 1 \\
\hline Cerebellar malformations & 1 & 1 \\
\hline Holoprosencephaly & 1 & 2 \\
\hline Cardiac defects & $6(66.7 \%)$ & $6(50.0 \%)$ \\
\hline Common atrioventricular cannal & 1 & 1 \\
\hline Undefined cardiac defect & 3 & 2 \\
\hline Cardiomegaly & 1 & \\
\hline Ventricular septal defect & 1 & \\
\hline Tetralogy of Fallot & 1 & 3 \\
\hline Urinary system defects & $0(0 \%)$ & $0(0 \%)$ \\
\hline Limb defects & $3(33.3 \%)$ & $5(41.7 \%)$ \\
\hline Club foot & 2 & \\
\hline Polidactyly & 3 & 5 \\
\hline \multicolumn{3}{|l|}{ Other structural defects } \\
\hline Omphalocele & 4 & 1 \\
\hline Ascites & 2 & \\
\hline Hydrothorax & 1 & \\
\hline Double buble & & 1 \\
\hline Hepatolegaly & 1 & \\
\hline Facial cleft & 1 & \\
\hline Micrognathia & & 2 \\
\hline \multicolumn{3}{|c|}{ Soft sonographic markers of chromosomal abnormalities } \\
\hline Increased nuchal translucency & $6(66.7 \%)$ & $0(0 \%)$ \\
\hline Increased nuchal fold & $3(33.3 \%)$ & $0(0 \%)$ \\
\hline Hyperechogenic bowel & $1(11.1 \%)$ & $0(0 \%)$ \\
\hline \multicolumn{3}{|l|}{ Placental abnormalities } \\
\hline Cystic placenta & $9(100 \%)$ & $0(0 \%)$ \\
\hline
\end{tabular}


Table 2 Characteristics and frequency of maternal complications in molecularly confirmed diandric and digynic triploid pregnancies

\begin{tabular}{lll}
\hline & $\begin{array}{l}\text { Diandric triploid pregnancies } \\
(n=9)\end{array}$ & $\begin{array}{l}\text { Digynic triploid pregnancies } \\
(n=12)\end{array}$ \\
\hline Maternal age (mean; years) & 31.1 (range 27.0-36.2) & 33.4 (range 26.3-46.1) \\
GA at genetic testing (g.w.) & 14.8 (range 11.7-17.6) & 18.0 (range 12.4-26.3) \\
Pregnancy duration (mean; g.w.) & 16.3 (range 14.1-19.6) & 19.3 (range 13.4-30.3) \\
Serum HCG level (mean; U/ml) & 979703.6 (range 382 261.0-1 636 562.0) & 5455.4 (range 1245.2-19 \\
& & $050.6)$ \\
Molar placenta & $100 \%$ & $0 \%$ \\
Thecalutein cysts in ovaries & $44.4 \%$ & $0 \%$ \\
Vaginal bleeding & $11.1 \%$ & $50.0 \%$ \\
Hyperemesis gravidarum & $44.4 \%$ & $0 \%$ \\
Symptomatic hyperthyreosis & $33.3 \%$ & $0 \%$ \\
Gestational hypertension & $22.2 \%$ & $0 \%$ \\
Preeclampsia & $0 \%$ & $0 \%$ \\
\hline
\end{tabular}

GA gestational age, g.w. gestational weeks

two and five cases, respectively, ended in intrauterine fetal demise. The follow-up of patients ranged between 6 months and 2 years. In none case GTN was observed. The mean time of normalization of beta-hCG in diandric cases was 84 days (range 11-142 days) and the control measurement after a month was within normal limits in all nine cases.

\section{Discussion}

We present a cohort of consecutive diandric and digynic triploid pregnancies with molecular confirmation that survived beyond 13 gestational weeks. To our knowledge this is the first assessment of maternal complications in such a group of patients. Second trimester triploidy is rare. Nevertheless the size of our study group enables us to draw some conclusions regarding the maternal risks associated with diandric and digynic triploidy, providing a basis for clinic and prenatal genetic counseling.

Molecular confirmation of parental origin was performed in only a few of the triploid pregnancies reported in the literature. Moreover, there is a possible bias provoked by the fact that only most severe cases are reported and their course is not representative for the whole group. The risk for maternal complications was also estimated in two bigger cohorts of triploid pregnancies, but without definitive distinction between diandric and digynic cases [19, 29]. Furthermore, there is a high discrepancy between the results presented in those studies.

The most typical complication of diandric triploid pregnancies is preeclampsia with an early onset before 20 gestational weeks $[1,3,4,8,10,11,13,15,19,23,24,26,28,29$, $33-35,37,38,41]$. 43 cases of hypertension or preeclampsia in apparently diandric triploidy were reported in the literature with the mean time of onset at 19 gestational weeks (range 14-39 gestational weeks) (Table 3).

The incidence of hypertension or preeclampsia was estimated for 4.3-35\% [19, 29]. In our cohort of diandric triploidies hypertension occurred in $22.2 \%$ of cases. No case of preeclampsia, eclampsia, HELLP syndrome or cardiomyopathy was noted. The relatively low incidence of hypertensive maternal complications and their less severe course in presented cohort may be attributed to the earlier prenatal diagnosis of diandric triploidy and termination of pregnancies before the onset of life-threatening maternal conditions. Previous cohort studies on the frequency of maternal complications in triploidy were published in the $90 \mathrm{~s}$, when the availability of ultrasound was much lower [19, 29]. In the era of broad application of the first trimester screening, the majority of diandric triploid cases may be detected before 14 gestational weeks, as was in our cohort [22]. In $66.7 \%$ of presented cases nuchal translucency exceeded $3.5 \mathrm{~mm}$ and in all cases with available free beta-hCG results in the first trimester, its level was very high, which resulted in a high patient-specific risk for trisomy 21 and further genetic testing that enabled appropriate diagnosis.

Despite the very high levels of total-hCG that were present in all our diandric cases, symptomatic hyperthyreosis occurred in only $33.3 \%$. We did not observe any relationship of thyreotoxicosis with the total-hCG level, which makes it difficult to predict. Hyperemesis gravidarum and thecalutein cysts were present in $44.4 \%$ of cases, respectively. In comparison, Jauniaux et al. reported a much lower rate of thecalutein cysts $(8.6 \%)$ and hyperemesis gravidarum $(5.7 \%)$. However, the authors included both diandric and digynic triploid cases to their study (1996). All of our diandric cases had molar changes in the placenta, while only $73 \%$ of molecularly confirmed 


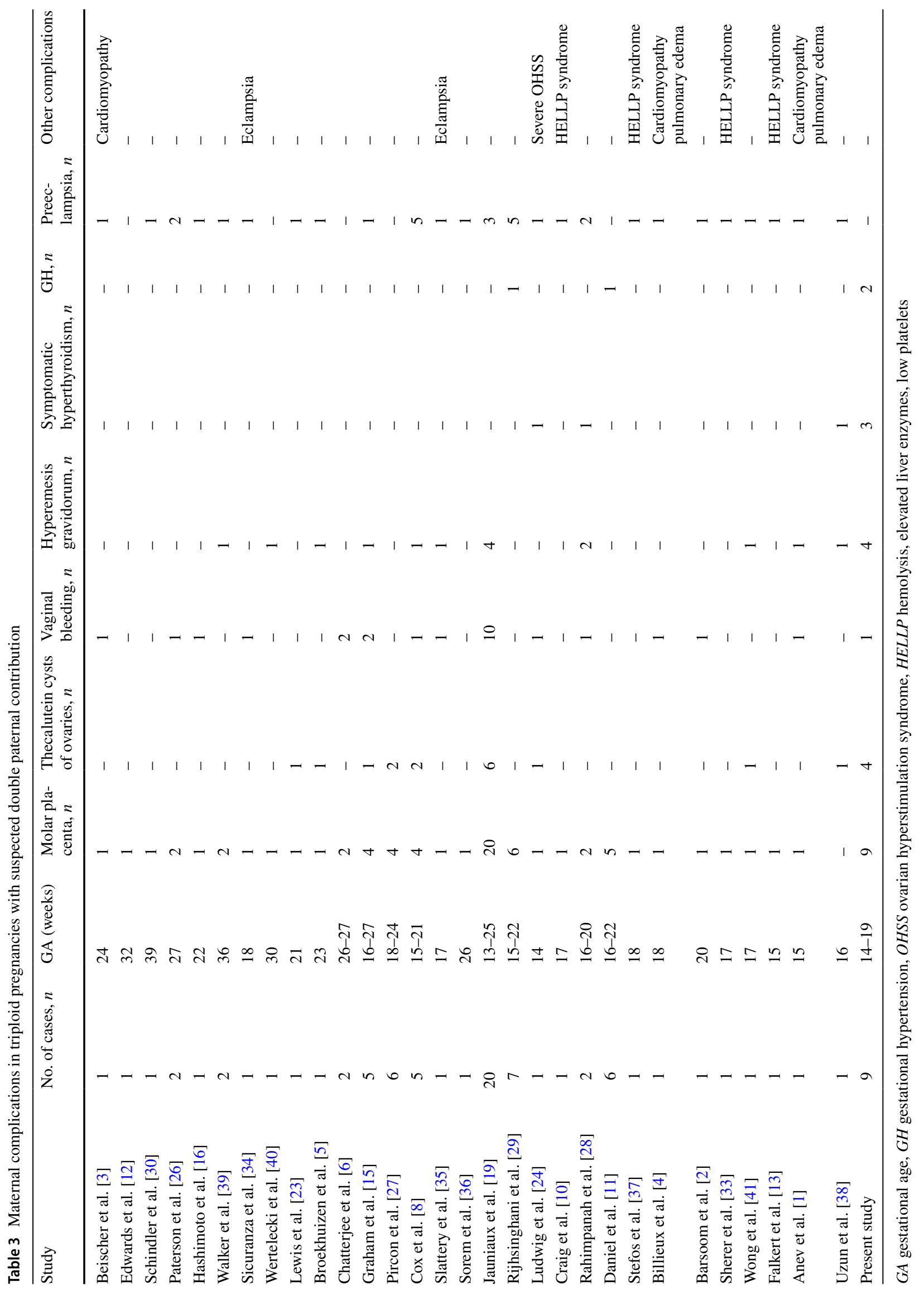


diandric cases described in the literature had molar appearance [21]. Bleeding was reported the most frequent complication in triploid pregnancies [19]. In our cohort bleeding occurred in $50.0 \%$ of digynic cases and only in $11.1 \%$ of diandric ones.

The frequency of GTN associated with triploid pregnancies beyond 13 weeks of gestation is difficult to establish. Single cases or small series are reported [7, 32]. In a huge group of 265 molecularly confirmed diandric triploid pregnancies with an over 6-month follow-up, no case of GTN was reported. However, gestational age of presented cases was not mentioned in the paper [31]. In our cohort of triploid pregnancies that survived beyond 13 gestational weeks (diandric as well as digynic), with a follow-up between 6 months and 2 years, no case of GTN was observed either. We concluded, therefore, that frequency of GTN after partial hydatidiform mole (diandric triploidy) may be much lower than previously reported [17]. However, the time of beta-hCG normalization after diandric triploid pregnancies may be very long-even exceeding 20 weeks as in one of our cases.

Even though our group consists of all consecutive cases of triploidy evaluated in a single institution, only cases with abnormal ultrasound presentation are reported, which is a limitation of our study. Nevertheless, to our knowledge this is the first analysis of maternal complications in molecularly confirmed diandric and digynic triploid pregnancies and our study may provide a basis for clinic and prenatal genetic counseling.

Author contribution DM: Project Development, Data Collection, Data analysis, Manuscript writing. JB: Data Collection, Data Analysis, Manuscript editing. AK: Data Collection, Manuscript editing. J. GZ: Project Development, Data Collection. KO: Data Collection. AR: Data Collection. G. MP: Revising for Important Intellectual Content. TR: Project Development, Data Collection.

Funding The study was partly funded by Centre of Postgraduate Medical Education in Warsaw (Project No. 501-1-21-27-18).

\section{Compliance with ethical standards}

Conflict of interest The authors declare that they have no conflict of interest.

Ethical approval All performed procedures were in accordance with the ethical standards of the Bioethics Committee of the Centre of Postgraduate Medical Education in Warsaw (Approval No. 82/PB/2017) and with the 1964 Helsinki Declaration and its later amendments.

Open Access This article is licensed under a Creative Commons Attribution 4.0 International License, which permits use, sharing, adaptation, distribution and reproduction in any medium or format, as long as you give appropriate credit to the original author(s) and the source, provide a link to the Creative Commons licence, and indicate if changes were made. The images or other third party material in this article are included in the article's Creative Commons licence, unless indicated otherwise in a credit line to the material. If material is not included in the article's Creative Commons licence and your intended use is not permitted by statutory regulation or exceeds the permitted use, you will need to obtain permission directly from the copyright holder. To view a copy of this licence, visit http://creativecommons.org/licenses/by/4.0/.

\section{References}

1. Anev I, Rajasri AG, Reddy K, Pillai M (2011) Triploidy without molar change presenting as severe pre-eclampsia and left ventricular failure at 15 weeks. J Obstet Gynaecol 31:659-660

2. Barsoom MJ, McEntaffer A, Fleming A, Nipper HC (2006) Marked abnormal quadruple screen in a patient with severe preeclampsia at 20 weeks with a triploid fetus. J Matern Fetal Neonatal Med 19(7):443-444

3. Beischer NA, Fortune DW, Fitzgerald MD (1967) Hydatidiform mole and coexistent fetus, both with triploid chromosome constitution. Br Med I 3:476

4. Billieux MH, Petignat P, Fior A et al (2004) Pre-eclampsia and peripartum cardiomyopathy in molar pregnancy: clinical implication for maternally imprinted genes. Ultrasound Obstet Gynecol 23:398-401

5. Broekhuizen FF, Elejalde R, Hamilton PR (1983) Earlyonset preeclampsia, triploidy and fetal hydrops. J Reprod Med 28:223-226

6. Chatterjee MS, Tejani NA, Vema UL et al (1983) Prenatal diagnosis of triploidy. Int J Gynecol Obstet 21:155

7. Cheung ANY, Khoo US, Lai CYL et al (2004) Metastatic trophoblastic disease after an initial diagnosis of partial hydatidiform mole. Cancer 100:1411-1417

8. Cox SM, Klein VR (1993) Partial molar pregnancy associated with severe pregnancy-induced hypertension. J Perinat 13:103-106

9. Coyle C, Short D, Jackson L et al (2018) What is the optimal duration of human chorionic gonadotrophin surveillance following evacuation of a molar pregnancy? A retrospective analysis on over 20,000 consecutive patients. Gynecol Oncol 148(2):254-257

10. Craig K, Pinette MG, Blackstone J, Chard R, Cartin A (2000) Highly abnormal maternal inhibin and $\beta$-human chorionic gonadotropin levels along with severe HELLP (hemolysis, elevated liver enzymes, and low platelet count) syndrome at 17 weeks' gestation with triploidy. Am J Obstet Gynecol 182:737-739

11. Daniel A, Wu Z, Bennetts B et al (2001) Karyotype, phenotype and parental origin in 19 cases of triploidy. Prenat Diagn 21:1034-1048

12. Edwards JH, Yuncken C, Rushton DJ, Richards S, Mittwoch U (1967) Three cases of triploidy in man. Cytogenetics 6:81

13. Falkert A, Yildiz A, Seelbach- Goebel B (2009) Partial mole with fetal triploidy as a cause for imminent HELLP-syndrome at 16 weeks of gestation. Arch Gynecol Obstet 279:423-425

14. Fergusson-Smith MA, Yates JRW (1984) Maternal age specific rates for chromosomal aberrations and factors influencing them: report of collaborative European study on 52,965 amniocenteses. Prenat Diagn 4:5-44

15. Graham JM, Rawnsley EF, Millard-Simmons G (1989) Triploidy: pregnancy complications and clinical findings in seven cases. Prenat Diagn 9:409-419

16. Hashimoto JS, Ling SH, Millerick JD, Kelley DJ (1973) Midtrimester preeclampsia associated with fetal triploidy: report of a case. Am J Obstet Gynecol 116:882-883

17. Hoffner L, Surti U (2012) The genetics of gestational trophoblastic disease: a rare complication of pregnancy. Cancer Genet 205:63-77

18. Jacobs PA, Szulman AE, Funkhouser J et al (1982) Human triploidy: relationship between parental origin of the additional 
haploid complement and development of partial hydatidiform mole. Ann Hum Genet 46:223-231

19. Jauniaux E, Brown R, Rodeck C, Nicolaides KH (1996) Prenatal diagnosis of triploidy during the second trimester of pregnancy. Obstet Gynecol 88:983-989

20. Jauniaux E, Brown R, Snijders RJM et al (1997) Early prenatal diagnosis of triploidy. Am J Obstet Gynecol 176:550-554

21. Joergensen MW, Niemann I, Rasmussen AA et al (2014) Triploid pregnancies: genetic and clinical features of 158 cases. Am J Obstet Gynecol 211:370.e1-19

22. Kagan KO, Anderson JM, Anwandter G, Neksasova K, Nicolaides $\mathrm{KH}$ (2008) Screening for triploidy by the risk algorithms for trisomies 21,18 and 13 at 11 weeks to 13 weeks and 6 days of gestation. Prenat Diagn 28:1209-1213

23. Lewis PE, Cefalo RC (1979) Triploidy syndrome with theca lutein cysts and severe preeclampsia. Am J Obstet Gynecol 133:110-111

24. Ludwig M, Gembruch U, Bauer O, Diedrich K (1998) Ovarian hyperstimulation syndrome (OHSS) in a spontaneous pregnancy with fetal and placental triploidy: information about the general pathophysiology of OHSS. Hum Reprod 13(8):2082-2087

25. McFadden DE, Kwong LC, Yam IYL, Langlois S (1993) Parental origin of triploidy in human fetuses: evidence for genomic imprinting. Hum Genet 92:465-469

26. Paterson WG, Hobson BM, Smart GE, Bain AD (1971) Two cases of hydatidiform degeneration of the placenta with fetal abnormality and triploid chromosome constitution. J Obstet Gynecol Br Commonw 78:136

27. Pircon RA, Porto M, Towers CV, Crade M, Gocke SE (1989) Ultrasound findings in pregnancies complicated by fetal triploidy. J Ultrasound Med 8:507-511

28. Rahimpanah F, Smoleniec J (2000) Partial mole, triploidy and proteinuric hypertension: two case reports. Aust N Z J Obstet Gynaecol 40:215-218

29. Rijhsinghani A, Yankowitz J, Strauss RA, Kuller JA, Patil S, Williamson RA (1997) Risk of preeclampsia in second-timester triploid pregnancies. Obstet Gynecol 90:884-888

30. Schindler AM, Mikamo K (1970) Triploidy in man. Cytogenetics 9:116
31. Scholz NB, Bolund L, Nyegaard M et al (2015) Triploidy-observations in 154 diandric cases. PLoS ONE 10(11):e0142545

32. Seckl MJ, Fisher RA, Salerno G et al (2000) Choriocarcinoma and partial hydatidiform moles. Lancet 356:36-39

33. Sherer DM, Dalloul M, Stimphil R et al (2006) Acute onset of severe hemolysis, elevated liver enzymes, and low platelet count syndrome in a patient with a partial hydatidiform mole at 17 weeks gestation. Am J Perinatol 23:163-166

34. Sicuranza BJ, Tisdall LH (1976) Hydatidiform mole and eclampsia with coexisting living fetus in the second trimester of pregnancy. Am J Obstet Gynecol 126:513

35. Slattery MA, Khong TY, Dawkins RR, Pridmore BR, Hague WM (1993) Eclampsia in association with partial molar pregnancy and congenital abnormalities. Am J Obstet Gynecol 169:1625-1627

36. Sorem KA, Shah DM (1995) Advanced triploid pregnancy and preeclampsia. South Med J 88:1144-1145

37. Stefos T, Plachouras N, Mari G, Cosmi I, Lolis D (2002) A case of partial mole and atypical type I triploidy associated with severe HELLP syndrome at 18 weeks' gestation. Ultrasound Obstet Gynecol 20:403-404

38. Uzun I, Pata O, Unlu C, Tokat F, Ozdemir M (2015) Uncommon presentation of triploidy: a case report. J Clin Diagn Res 9(10):QD01-QD02

39. Walker S, Andrews J, Gregson NM, Gault W (1973) Three further cases of triploidy in man surviving to birth. J Med Genet 10:135-141

40. Wertelecki W, Graham JM, Sergovich FR (1976) The clinical syndrome of triploidy. Obstet Gynecol 47:69-76

41. Wong LFA, Stuart B, Gleeson N (2007) Triploidy partial mole and proteinuric hypertension. J Obstet Gynaecol 27:424-425

Publisher's Note Springer Nature remains neutral with regard to jurisdictional claims in published maps and institutional affiliations. 